\title{
Some 3-Amino and 3-Substituted-amino Derivatives of $\alpha^{4}$-Norpyridoxol ${ }^{\dagger}$
}

\author{
Mitsuru Kataoka, Toshiaki Sakamoto, Fumiko Saito \\ and Yasuhiro MorisAwa
}

Central Research Laboratories, Sankyo Co., Ltd., Shinagawa-ku, Tokyo

Received February 10, 1975

\begin{abstract}
Some analogs of $\alpha^{4}$-norpyridoxol in which 3-hydroxyl group is replaced by amino and substituted-amino groups have been prepared and evaluated for anticoccidal activity. 3Aroylamino analogs of $\alpha^{4}$-norpyridoxol have some coccidiostatic effect towards Eimeria tenella.
\end{abstract}

As a part of our investigations on the structural modifications of $\alpha^{4}$-norpyridoxol (1) ${ }^{1)}$ and 4-deoxypyridoxol (2, 4-DOP), ${ }^{2)}$ a new type of anticoccidial drugs, we have prepared 3-amino derivatives of $\alpha^{4}$-norpyridoxol and 4-DOP.<smiles></smiles>

FIG. 1.

3-Amino-6-chloro-5-cyano-2-methylpyridine, obtained from 5-cyano-2-methylpyridine by the procedure of Perez-Median et $a l^{3}{ }^{3}$ was acylated with acyl chlorides in pyridine. The resultant 3-aroylamino derivatives $(\mathbf{2} \mathbf{a}, \mathbf{b})$ were hydrogenated in the presence of palladium on carbon to give 5-aminomethyl-3-aroylamino-2-methylpyridines $(\mathbf{3 a}, \mathbf{b})$, while a similar attempt to convert the 3 -acetylamino5-cyano-derivative (2c) to the corresponding 5-aminomethyl compound (3) yielded only 3amino-5-aminomethyl-2-methylpyridine (6). Diazotization of the primary amine $(\mathbf{3 a}, \mathbf{b})$, followed by hydrolysis of the diazonium salt gave 3-aroylamino-5-hydroxymethyl-2-methylpyridines $(\mathbf{4 a}, \mathbf{b})$. Treatment of benzoylamino derivative (4a) with hydrochloric acid gave a good yield of 3-amino-5-hydroxymethyl-2-

$\dagger$ Studies on Anticoccidial Agents. Part V. For paper Part IV of this series see Y. Morisawa, M. Kataoka, T. Watanabe, N. Kitano and T. Matsuzawa, Agr. Biol. Chem., 39, 1275 (1975). methylpyridine (5). Greene et al. ${ }^{4}$ has reported the synthesis of 3-amino-5-hydroxymethyl2,4-dimethylpyridine, but did not describe any biological activity.

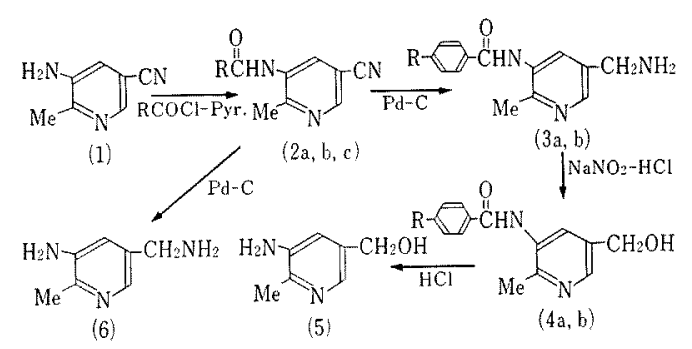

a; $\mathrm{R}=\mathrm{Ph}, \mathrm{b} ; \mathrm{R}=p$-Methoxyphenyl, $c ; \mathrm{R}=\mathrm{Ac}$

FIG. 2.

3-Amino - 5-hydroxymethyl-2, 4-dimethylpyridine and 3-amino-5-hydroxymethyl-2-methylpyridine (5) as well as the intermediates $(3,4)$ leading to $(5)$ have been tested against Eimeria acervulina and E. tenella. The 3amino analog of 4-DOP exhibited moderate anticoccidial activity against $E$. acervulina at $200 \mathrm{ppm}$ in feed but was less active than $\alpha^{4}$ norpyridoxol. 3-Aroylamino compounds (3, 4) have some coccidiostatic effect towards E. tenella.

\section{EXPERIMENTAL}

Melting points were uncorrected. IR spectra were obtained with Perkin-Elmer 221 and JASCO IRA-2 spectrometers and NMR spectra were recorded with a Varian A-60 spectrometer using TMS as an internal standard. Typical experimental procedures are de- 
scribed for the preparation of compounds (2, 3 and 4).

3-Benzoylamino-6-chloro-5-cyano-2-methylpyridine (2a) Benzoyl chloride $(5.6 \mathrm{~g})$ was added dropwise to a solution of 3-amino-6-chloro-5-cyano-2-methylpyridine $(1,5.6 \mathrm{~g})$ in pyridine $(70 \mathrm{ml})$. The mixture was stirred at room temperature for $16 \mathrm{hr}$, poured into ice-water and extracted with $\mathrm{CHCl}_{3}$. The extract was washed with water, dried $\left(\mathrm{Na}_{2} \mathrm{SO}_{4}\right)$ and the solvent was removed to leave a crystalline product. Recrystallization from EtOAc- $n$-hexane gave $7.95 \mathrm{~g}$ of $(2 \mathrm{a}), \mathrm{mp} 205 \sim 206^{\circ} \mathrm{C}$. IR $\nu_{\max }^{\mathrm{Nujol}} \mathrm{cm}^{-1}: 3360,2250,1680$. Anal. Found: C, $62.00 ; \mathrm{H}, 3.67 ; \mathrm{N}, 15.51 ; \mathrm{Cl}, 13.23$. Calcd. for $\mathrm{C}_{14} \mathrm{H}_{10^{-}}$ $\mathrm{N}_{3} \mathrm{OCl}: \mathrm{C}, 61.80 ; \mathrm{H}, 3.68 ; \mathrm{N}, 15.35 ; \mathrm{Cl}, 13.08$.

3-p-Methoxybenzoylamino-6-chloro-5-cyano-2-methylpyridine (2a)

Mp $238 \sim 240^{\circ} \mathrm{C} . \quad$ IR $\nu_{\max }^{\text {Nujol }} \mathrm{cm}^{-1}: 3350,2230,1670$. Anal. Found: C, $59.79 ; \mathrm{H}, 3.91 ; \mathrm{N}, 13.83 ; \mathrm{Cl}, 11.58$. Calcd. for $\mathrm{C}_{15} \mathrm{H}_{12} \mathrm{~N}_{3} \mathrm{O}_{2} \mathrm{Cl}: \mathrm{C}, 59.71 ; \mathrm{H}, 4.01 ; \mathrm{N}, 13.93$; $\mathrm{Cl}, 11.75$.

3-Acetylamino-6-chloro-5-cyano-2-methylpyridine (2c)

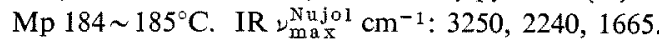
Anal.Found: C, 51.65; H, 3.84; N, 20.25; Cl, 16.69. Calcd. for $\mathrm{C}_{9} \mathrm{H}_{8} \mathrm{~N}_{3} \mathrm{OCl}: \mathrm{C}, 51.56 ; \mathrm{H}, 3.85 ; \mathrm{N}, 20.05$; $\mathrm{Cl}, 16.91$.

\section{5-Aminomethyl-3-benzoylamino-2-methylpyridine $2 \mathrm{HCl}$} (3a)

To a suspension of preactivated $10 \% \mathrm{Pd}-\mathrm{C}$ catalyst $(2.8 \mathrm{~g})$ in $\mathrm{MeOH}(60 \mathrm{ml})$ containing conc. $\mathrm{HCl}(5 \mathrm{ml})$ was added a solution of 3-benzoylaminopyridine (2a, $2.8 \mathrm{~g})$ in $\mathrm{MeOH}(200 \mathrm{ml})$. The mixture was shaken in $\mathrm{H}_{2}$ atmosphere for $8 \mathrm{hr}$. After separation of the catalyst, the filtrate was evaporated into dryness to leave a colorless crystalline product $(2.9 \mathrm{~g})$, which was recrystallized from $\mathrm{MeOH}$ to give (3a), $\mathrm{mp} 230 \sim 250^{\circ} \mathrm{C}$ (indefinite). IR $\nu_{\max }^{\mathrm{Nujol}} \mathrm{cm}^{-1}: \quad 3025,1680$. NMR $\delta_{\mathrm{TMS}}^{\mathrm{DMSO}-\mathrm{D}_{6}}: 2.78$ $\left(3 \mathrm{H}, \mathrm{s}, \mathrm{C}_{2}-\mathrm{Me}\right), 4.25\left(2 \mathrm{H}\right.$, broad, $\left.\mathrm{C}_{5}-\mathrm{CH}_{2}\right), 7.41 \sim 7.74$ $(3 \mathrm{H}$, aromatic $\mathrm{H}), 8.01 \sim 8.26(2 \mathrm{H}$, aromatic $\mathrm{H}), 8.63$ (1H, d, $J=1.5 \mathrm{~Hz}), 8.76(1 \mathrm{H}, \mathrm{d}, J=1.5 \mathrm{~Hz})$. Anal. Found: $\mathrm{C}, 53.41 ; \mathrm{H}, 5.40 ; \mathrm{N}, 13.28 ; \mathrm{Cl}, 22.36$. Calcd. for $\mathrm{C}_{14} \mathrm{H}_{17} \mathrm{~N}_{3} \mathrm{OCl}_{2}: \mathrm{C}, 53.52 ; \mathrm{H}, 5.45 ; \mathrm{N}, 13.37 ; \mathrm{Cl}, 22.56$.

\section{5-Aminomethyl-3-p-methoxybenzoylamino-2-methylpyri- dine $2 \mathrm{HCl}$ (3b)}

Mp $250^{\circ} \mathrm{C}$. IR $v_{m a s}^{\text {Nujol }} \mathrm{cm}^{-1}: 1680$. NMR $\delta_{\text {TMS }}^{\text {DMSO-d }}$ : $2.78\left(3 \mathrm{H}, \mathrm{s}, \mathrm{C}_{2}-\mathrm{Me}\right), 3.85(3 \mathrm{H}, \mathrm{s}, \mathrm{OMe}), 4.26(2 \mathrm{H}$, broad, $\left.\mathrm{C}_{5}-\mathrm{CH}_{2}\right), 7.07(2 \mathrm{H}, \mathrm{d}, J=8.5 \mathrm{~Hz}$, aromatic $\mathrm{H})$, $8.13(2 \mathrm{H}, \mathrm{d}, J=8.5 \mathrm{~Hz}$, aromatic $\mathrm{H}), 8.73(1 \mathrm{H}, \mathrm{d}$, $J=1.5 \mathrm{~Hz}), 8.85(1 \mathrm{H}, \mathrm{d}, J=1.5 \mathrm{~Hz})$. Anal. Found: C, 52.19; H, 5.72; N, 12.06; Cl, 20.61; Calcd. for $\mathrm{C}_{15} \mathrm{H}_{19} \mathrm{~N}_{3} \mathrm{O}_{2} \mathrm{Cl}_{2}: \mathrm{C}, 52.34 ; \mathrm{H}, 5.56 ; \mathrm{N}, 12.21, \mathrm{Cl}, 20.60$.

3-Amino-5-aminomethyl-2-methylpyridine $2 \mathrm{HCl}$ (6)

A solution of 3-acetylamino-5-cyano-2-methyl- pyridine $(2 \mathrm{c}, 3 \mathrm{~g})$ in $\mathrm{MeOH}(160 \mathrm{ml})$ containing conc. $\mathrm{HCl}(9 \mathrm{ml})$ was hydrogenated in the presence of $10 \%$ Pd-C ( $4 \mathrm{~g})$. After separation of the catalyst, the filtrate was concentrated to give a pale brown crystalline product. Recrystallization from $\mathrm{MeOH}$ afforded $1.99 \mathrm{~g}$ of the diamino derivative (6), mp $295 \sim 296^{\circ} \mathrm{C}$ (dec) (lit. $^{31}$ dec. $295 \sim 297^{\circ} \mathrm{C}$ ). Anal. Found: C, 39.87; H, 6.34; $\mathrm{N}, 19.96 ; \mathrm{Cl}, 33.70$. Calcd. for $\mathrm{C}_{7} \mathrm{H}_{13} \mathrm{~N}_{3} \mathrm{Cl}_{2}$ : C, 39.99; H, 6.24; N, 20.01; Cl, 33.76.

\section{3-Benzoylamino-5-hydroxymethyl-2-methylpyridine $\mathrm{HCl}$ (4a)}

An aqueous solution $(16 \mathrm{ml})$ of $\mathrm{NaNO}_{2}(1.0 \mathrm{~g})$ was added at $80^{\circ} \mathrm{C}$ in $20 \mathrm{~min}$ to a solution of 5-aminomethyl compound (3a, $3.2 \mathrm{~g}$ ) in $0.5 \mathrm{~N} \mathrm{HCl}$ solution $(20 \mathrm{ml})$. The mixture was stirred at $80^{\circ} \mathrm{C}$ for $1 \mathrm{hr}$, the solvent was removed to leave a crystalline residue, which was extracted with absolute EtOH several times. The extract was concentrated into a small volume and addition of EtOAc afforded a crystalline product. Recrystallization from the same solvent mixture gave $1.85 \mathrm{~g}$ of (4a), $m p 175 \sim 200^{\circ} \mathrm{C}$ (indefinite). $\operatorname{IR} \nu_{\mathrm{max}}^{\mathrm{Nujjol}} \mathrm{cm}^{-1}$ : 3325,1685 . NMR $\hat{o}_{\text {TiSS }}^{\mathrm{D}_{2} \mathrm{O}}: 2.72\left(3 \mathrm{H}, \mathrm{s}, \mathrm{C}_{2}-\mathrm{Me}\right), 4.87$ $\left(2 \mathrm{H}, \mathrm{s}, \mathrm{C}_{5}-\mathrm{CH}_{2}\right), 7.55 \sim 7.80(3 \mathrm{H}, \mathrm{m}$, aromatic $\mathrm{H})$, $7.80 \sim 8.10(2 \mathrm{H}, \mathrm{m}$, aromatic $\mathrm{H}), 8.55(2 \mathrm{H}$, broad $\mathrm{s}$, $\mathrm{C}_{4}$ and $\left.\mathrm{C}_{6}-\mathrm{H}\right)$. Anal. Found: $\mathrm{C}, 60.31 ; \mathrm{H}, 5.47$; $\mathrm{N}, 10.25 ; \mathrm{Cl}, 12.77$. Calcd. for $\mathrm{C}_{14} \mathrm{H}_{15} \mathrm{~N}_{2} \mathrm{O}_{2} \mathrm{Cl}: \mathrm{C}$, $60.33 ; \mathrm{H}, 5.42 ; \mathrm{N}, 10.05 ; \mathrm{Cl}, 12.72$.

\section{3-p-Methoxybenzoylamino-5-hydroxymethyl-2-methyl- pyridine $\mathrm{HCl}$ (4b)}

$\mathrm{Mp} 200 \sim 212^{\circ} \mathrm{C}$ (indefinite) on recrystallization from EtOH-ether. IR $\nu_{\max }^{\mathrm{Nujol}} \mathrm{cm}^{-1}: 3350,3225,1670$. NMR $\delta_{\mathrm{TMS}}^{\mathrm{DMSO}-\mathrm{d}_{6}}: 2.75\left(3 \mathrm{H}, \mathrm{s}, \mathrm{C}_{2}-\mathrm{Me}\right), 3.85$ (3H, s, OMe), 4.70 $\left(2 \mathrm{H}, \mathrm{s}, \mathrm{C}_{5}-\mathrm{CH}_{2}\right), 7.06(2 \mathrm{H}, \mathrm{d}, J=8.5 \mathrm{~Hz}$, aromatic $\mathrm{H})$, $8.10(2 \mathrm{H}, \mathrm{d}, J=8.5 \mathrm{~Hz}$, aromatic $\mathrm{H}), 8.53\left(2 \mathrm{H}, \mathrm{C}_{4}\right.$ and $\mathrm{C}_{6}-\mathrm{H}$ ). Anal. Found: C, 58.60; $\mathrm{H}, 5.48 ; \mathrm{N}, 9.07$; $\mathrm{Cl}, 11.66$. Calcd. for $\mathrm{C}_{15} \mathrm{H}_{17} \mathrm{~N}_{2} \mathrm{O}_{3} \mathrm{Cl}: \mathrm{C}, 58.35, \mathrm{H}, 5.55$; $\mathrm{N}, 9.07 ; \mathrm{Cl}, 11.48$.

\section{3-Amino-5-hydroxymethyl-2-methylpyridine $\mathrm{HCl}(\mathbf{5})$}

A solution of 3-benzoylamino compound $(4 \mathrm{a}, 1.3 \mathrm{~g})$ in $5 \mathrm{~N} \mathrm{HCl}(60 \mathrm{ml})$ was refluxed for $5 \mathrm{hr}$, cooled and shaken with ether. The aqueous layer separated was concentrated into dryness to give a colorless product, which was recrystallized from $\mathrm{MeOH}$ to afford $0.63 \mathrm{~g}$ of 5, mp 232 236 2 . IR $\nu_{\mathrm{max}}^{\mathrm{Nujol}} \mathrm{cm}^{-1}: 3400,3325$, 3210. NMR $\delta_{\mathrm{TMS}}^{\mathrm{DMSO}-\mathrm{d}_{6}}: 2.53\left(3 \mathrm{H}, \mathrm{s}, \mathrm{C}_{2}-\mathrm{Me}\right), 4.55(2 \mathrm{H}$, s, $\left.\mathrm{C}_{5}-\mathrm{CH}_{2}\right), 7.60(1 \mathrm{H}, \mathrm{d}, J=1.5 \mathrm{~Hz}), 7.77(1 \mathrm{H}, \mathrm{d}, J=$ $1.5 \mathrm{~Hz}$ ). Anal. Found: C, 47.99; H, 6.51; N, 15.94; Cl, 20.47. Calcd. for $\mathrm{C}_{7} \mathrm{H}_{11} \mathrm{~N}_{2} \mathrm{OCl}: \mathrm{C}, 48.14 ; \mathrm{H}, 6.35$; $\mathrm{N}, 16.04 ; \mathrm{Cl}, 20.30$.

Acknowledgement. We wish to express our gratitude to Dr. Ko Arima, Director of these Laboratories and to Dr. K. Murayama, Assistant Director, for their 
encouragement and discussion. We are also indebted to Messrs. N. Kitano and T. Matsuzawa for the evaluation of the anticoccidial activity of these compounds.

\section{REFERENCES}

1) Y. Morisawa, M. Kataoka, T. Watanabe, N. Kitano and T. Matsuzawa, J. Med. Chem., 17,
1235 (1974).

2) Y. Morisawa, M. Kataoka, T. Watanabe, N. Kitano and T. Matsuzawa, J. Med. Chem., 17, 1083 (1974).

3) L. A. Perez-Medina, R. P. Mariella and S. M. McElvain, J. Am. Chem. Soc., 69, 2574 (1947).

4) J. L. Greene, Jr., and J. A. Montgomery, J. Med. Chem., 7, 17 (1964). 